\title{
COMPARISON OF LISS-IV MX AND LISS-III+LISS-IV MERGED DATA FOR CLASSIFICATION OF CROPS
}

\author{
R. Hebbar ${ }^{a}$ and M.V.R. Sesha Sai ${ }^{b}$ \\ ${ }^{a}$ Regional Remote Sensing Centre -South, NRSC/ISRO, Marathahalli, Bengaluru, India - 560037 (hebbar_kr@nrsc.gov.in) \\ ${ }^{b}$ National Remote Sensing Centre, ISRO, Balanagar, Hyderabad, India - 500037 (seshasai_mvr@nrsc.gov.in)
}

Technical Commission VIII

KEY WORDS: Resourcesat-1, data fusion, quality parameters, classification, accuracy, crops

\begin{abstract}
:
Resourcesat-1 satellite with its unique capability of simultaneous acquisition of multispectral images at different spatial resolutions (AWiFS, LISS-III and LISS-IV MX / Mono) has immense potential for crop inventory. The present study was carried for selection of suitable LISS-IV MX band for data fusion and its evaluation for delineation different crops in a multi-cropped area. Image fusion techniques namely intensity hue saturation (IHS), principal component analysis (PCA), brovey, high pass filter (HPF) and wavelet methods were used for merging LISS-III and LISS-IV Mono data. The merged products were evaluated visually and through universal image quality index, ERGAS and classification accuracy. The study revealed that red band of LISS-IV MX data was found to be optimal band for merging with LISS-III data in terms of maintaining both spectral and spatial information and thus, closely matching with multispectral LISS-IVMX data. Among the five data fusion techniques, wavelet method was found to be superior in retaining image quality and higher classification accuracy compared to commonly used methods of IHS, PCA and Brovey. The study indicated that LISS-IV data in mono mode with wider swath of $70 \mathrm{~km}$ could be exploited in place of 24km LISS-IVMX data by selection of appropriate fusion techniques by acquiring monochromatic data in the red band.
\end{abstract}

\subsection{INTRODUCTION}

Spectral, spatial and temporal resolution information from multi-sensor satellite data can be exploited for mapping and monitoring of natural resources. The synergetic use of multisensor data utilizing various fusion techniques gained considerable attention of researchers (Pohl and Van Genderen, 1998). Several studies have been carried out for evaluating data fusion techniques (Wang et al., 2005 and Colditz et al., 2006). The most commonly used data fusion techniques are intensity hue saturation (IHS), principal component analysis (PCA) and Brovey. Several authors have used the multi resolution analysis and wavelet transforms to introduce the spatial information into the spectral bands (Kumar et al., 2000 and Chen Yunhao et al., 2006). Evaluation of these data fusion techniques for merging IRS L-3 and PAN data have been investigated (Mohanty and Majumdar, 2003 and Ray, 2004).

The major limitation for successful utilization of multi sensor data is lack of simultaneously acquired data. Resourcesat-1 satellite has the unique capability of simultaneously acquiring multispectral images at 56, 24 and $6 \mathrm{~m}$ spatial resolution. LISS-IV mode operates in multispectral mode with $23 \mathrm{~km}$ swath while the Mono mode operates in panchromatic mode with a wider swath of $70 \mathrm{~km}$ resolution. The performance of each LISS-IV MX band for producing band-sharpened multispectral images was evaluated and observed that red band is optimal for merging with L-3 data (Kumar et al., 2006). The fused multispectral data has potential for agricultural applications especially horticultural crops as these crops are cultivated under heterogeneous systems and scattered, requiring high spatial resolution data.

\footnotetext{
* Corresponding Author
}

The potential use of merged data for classification of agricultural and horticultural crops is not studied in detail. The present study was carried out to merge LISS-III and LISS-IV mono data for crop discrimination with the objectives of (a) Evaluation of different data fusion techniques and (b) Selection of optimal monochromatic band of LISS-IV MX data for merging with LISS-III data for classification of crops.

\subsection{MATERIALS AND METHODS}

\subsection{Study Area}

The study area is located in East Godavari district of Andhra Pradesh, India lying between $81^{\circ} 49^{\prime}$ to $81^{\circ} 55^{\prime}$ E longitudes and $16^{\circ} 57^{\prime}$ to $17^{\circ} 03^{\prime} \mathrm{N}$ latitudes. The study area is characterized by heterogeneous cropping pattern with multicrops and horticultural plantations. Coconut, oil palm and cashew are the important horticultural crops of the study area while rice and sugarcane are important field crops.

Among horticultural crops, coconut and oil palm are cultivated in larger land holdings which are closely spaced fully covering the soil background while cashew is cultivated with wider spacing and scattered throughout the study area. The ground truth information on different crops cultivated in the study area was used for generating training samples for the supervised classification and accuracy assessment. Topographical maps and Global Positioning System (GPS) were utilized for accurately locating the ground truth sites.

\subsection{Satellite Data}

Resourcesat-1 LISS-III data (L-3) with $23.5 \mathrm{~m}$ spatial resolution and LISS-IV MX (L-4) data of $5.8 \mathrm{~m}$ spatial resolution acquired simultaneously on March 05, 2008 was 
used in the study. The characteristics of L-3 and L-4 sensors are provided in Table-1. L-4 camera operates in two modes; mono and multi-spectral mode. In multi-spectral mode, the data is collected in three spectral bands viz., green, red and NIR with $24 \mathrm{~km}$ swath, while in the mono mode the data is nominally collected in the red band with wider swath of 70 $\mathrm{Km}$. The L-4 data has an additional MIR band which was not used in the study. A sub image of the datasets covering 2000 X 2000 pixels was analysed in the study.

\begin{tabular}{|l|c|c|c|}
\hline \multicolumn{1}{|c|}{ Details } & L-3 & L-4 MX & L-4 Mono \\
\hline Spectral & $0.52-0.59$ & $0.52-0.59$ & \\
Resolution & $0.62-0.68$ & $0.62-0.68$ & Red band \\
(um) & $0.76-0.86$ & $0.76-0.86$ & \\
& $1.55-1.70$ & & \\
\hline IFOV (m) & 23.5 & 5.8 & 5.8 \\
\hline Swath (Km) & 141 & 24 & 70 \\
\hline Radiometry & \multicolumn{3}{|c|}{ Best 7 out of 10 bits } \\
\hline Repetivity & \multicolumn{3}{|c|}{ 24 days } \\
\hline
\end{tabular}

Table-1: Specifications of Resourcesat-1 sensors

\subsection{Data fusion}

L-3 and L-4 data were co-registered with help of adequate number of Ground Control Points (GCP) in order to achieve sub pixel registration accuracy and resampled using neighbourhood technique for data fusion.

In the present study, five fusion techniques namely Brovey (Pohl and Van Genderen, 1998), Intensity Hue and Saturation (Welch and Ehlers, 1987), Principal Component Analysis (Richards, 1986), High Pass Filter (Chavez et al., 1991) and Wavelet (King and Wang, 2001 and AS Field Guide, 2003) were used for data merging. Green, red and NIR bands of L-4MX were independently merged with L-4 data resulting into a total of 15 multi-colour images. These resultant images generated using green, red and NIR bands of L-4 data were referred as G-MX, R-MX and NIR-MX, respectively, as given in Fig-1.

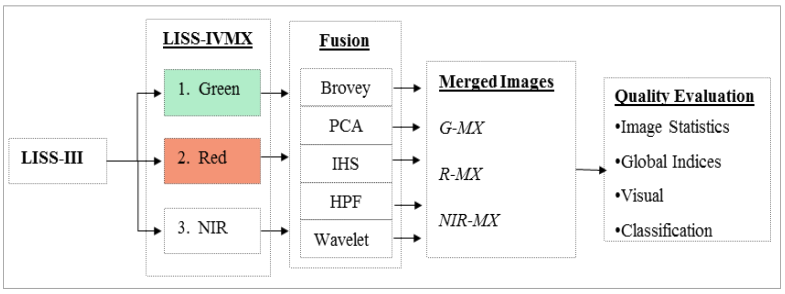

Fig-1: Steps involved in the data analysis

\subsection{Image quality evaluation}

The merged products were compared with reference to L-3 and L-4 data for assessing image quality and selection of optimal monochromatic bands among the three bands of L-4 data. The quality parameters are grouped into four categories

- Image statistics: band-wise mean, standard deviation (SD) and correlation coefficient were analysed for the entire scene and important crop classes.

- Visual interpretation: visual interpretation of merged product in terms of manifestation of crops and geometric features such as roads and field boundaries
- Global measures: ERGAS index (Wald et al, 1997), Universal Image Quality Index (Wang and Bovik, 2002)

\section{○ Classification accuracy}

Wald et al., 1997 proposed image quality assessment index, ERGAS, which incorporates multi-band characteristics for comparing the image quality as given in equation (1).

$$
E R G A S=100 \frac{h}{l} \sqrt{\frac{1}{N} \sum_{k=1}^{N} \frac{R M S E\left(B_{k}\right)^{2}}{\left(M_{k}\right)^{2}}}
$$

where, $\mathrm{h}$ and $\mathrm{l}$ are low and high resolution data (6/24 for L-4 / L-3 data), $\mathrm{N}$ is number of bands, $\operatorname{RMSE}\left(\mathrm{B}_{\mathrm{k}}\right)$ is root mean square error of band $\mathrm{k}$ and $\mathrm{M}_{\mathrm{k}}$ is mean of band $\mathrm{k}$. Low values of ERGAS indicate better image quality indicating better performance of fusion method. The ERGAS index was generated for L-4 and the merged products separately and compared with the reference L-3 image. The universal image quality index $(\mathrm{Q})$ which is applicable to the each single band of the image is given equation (2).

$$
Q n=\frac{4 \sigma_{x y} \bar{X} \bar{Y}}{\left(\sigma_{X}^{2}+\sigma_{X}^{2}\right)\left(\bar{X}^{2}+\bar{Y}^{2}\right)}
$$

Where $\sigma_{\mathrm{XY}}$ is the covariance between $\mathrm{X}$ and $\mathrm{Y}, \bar{X}$ and $\bar{Y}$ their means, and $\sigma_{X} \& \sigma_{Y}$ their variances, respectively. The $Q$ index is a unique number with a dynamic range of +1 to -1 with higher $\mathrm{Q}$ value indicates lower radiometric distortions and better image quality.

\subsection{Classification}

The classification accuracy of merged products was also evaluated for delineation of various land cover classes including agricultural and horticultural crops. Training areas were specified for eight broad land cover classes viz., coconut, cashew, oil palm, rice, sugarcane, fallow, urban and water. The class separability among the training areas was studied by generating two band class ellipses and transformed divergence matrices (TD) and classified using maximum likelihood classification algorithm. The post classification accuracy assessment was carried out by generating confusion matrix and computing accuracy parameters viz., overall accuracy and kappa coefficient for evaluation of data fusion techniques for thematic mapping.

\subsection{Results and Discussion}

\subsection{Image quality parameters}

Image statistics in terms of mean and SD of the merged products were compared with L-3 and L-4MX data and depicted in Fig-2. The mean digital numbers (DN) of L-3 and L-4 data was comparable however, L-4 data showed higher SD due to better spatial resolution. Among different fusion techniques, the mean and SD of wavelet merged product was closer to the L-3 data indicating retention of original image quality without adversely affecting the spectral characteristics. Brovey transform considerably reduced both mean and SD and conversely, PCA method resulted in higher mean and SD indicating radiometric distortions in these transformations. The training area statistics of major crop class viz., coconut, cashew, oil palm and rice also showed similar trend. Ray, 2004 showed similar results when different fusion techniques were applied to IRS-1D L-3 and PAN data. 

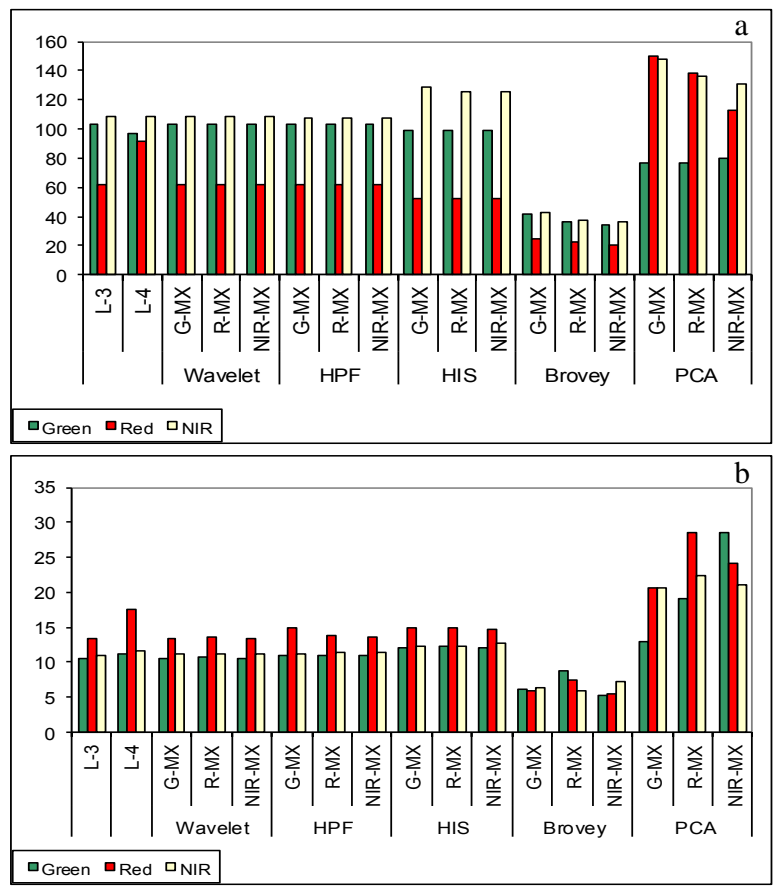

Fig-2: Mean (a) and standard deviation (b) of L-3, L-4 and merged images

The correlation coefficient (CC) between L-3 with corresponding L-4 and merged image bands is shown in Fig3. Higher CC was observed between the three L-3 and corresponding L-4 bands with red band showing highest CC of 0.91. HPF and wavelet methods have shown higher $\mathrm{CC}$ for G-MX, R-MX and NIR-MX compared to other methods indicating that these methods are superior for data merging while retaining original image spectral quality. PCA and IHS methods have shown lowest correlation among the five techniques indicating larger deviations in the radiometry of the merged images. Further, the intensity and the first principal component had shown significant correlations with red (0.81 and 0.86) and green (0.78 and 0.83) bands of L-4 data and very poor correlation with near infrared band $(0.26$ and 0.19 ). This indicated the suitability of red or green band for generating the fused images.

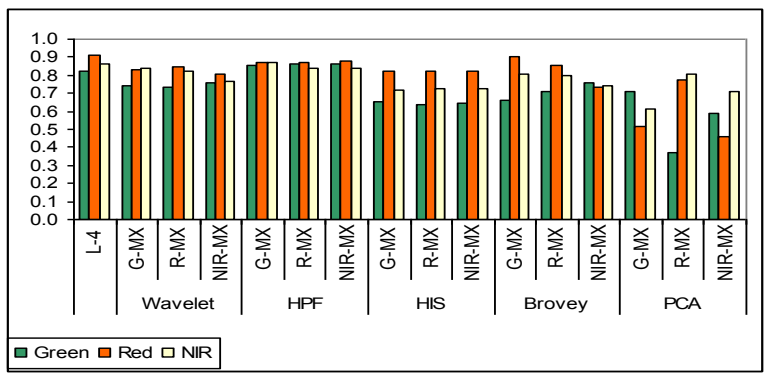

Fig-3: Correlation coefficient (CC) of L-3 with L-4 and merged images

The global image quality index ERGAS was calculated for the five merged images using green, red and NIR bands of L4 data independently and is presented in Table-2. The ERGAS values for the L-3 and L-4 were 3.72 and 3.39 , respectively. The table indicates that wavelet and HPF methods showed lowest ERGAS among the five methods and were close to the reference L-3 data. IHS, Brovey and PCA methods showed higher ERGAS values indicating image distortions and unsuitable for data fusion. Comparative performance of three mono bands of L-4 data for fusion using ERGAS index alone indicated that G-MX and R-MX performed better scoring and wavelet was the best method for data merging. Kumar et al., 2006 also observed lowest ERGAS with G-MX combination. The results suggests that either green or red band of L-4MX data could be used for data merging due to strong correlation (0.96) between R-MX and G-MX with similar dynamic range. However, the image manifested well with R-MX.

\begin{tabular}{|c|l|r|r|r|}
\hline S. N. & Fusion Method & G-MX & R-MX & \multicolumn{1}{c|}{ NIR-MX } \\
\hline 1 & Wavelet & 3.79 & 3.80 & 3.84 \\
\hline 2 & HPF & 3.85 & 3.88 & 3.89 \\
\hline 3 & HIS & 4.67 & 4.71 & 4.67 \\
\hline 4 & Brovey & 5.07 & 5.31 & 6.38 \\
\hline 5 & PCA & 5.86 & 7.03 & 7.97 \\
\hline
\end{tabular}

Table-2: ERGAS index for different fusion methods

The Q index calculated for each single band of L-4 and merged products using L-3 as the reference data is presented in Table-3. A comparison of $\mathrm{Q}$ index for different fusion methods indicate that wavelet and HPF transform showed higher mean Q indices, which were very close to L-4 image indicating the retention of spectral properties of the original multispectral L-4 data by these two of the five tested methods of data fusion. It was also observed that the mean $\mathrm{Q}$ index for L-3 data was also similar L-4MX indicating the spectral similarity.

\begin{tabular}{|c|c|c|c|c|c|}
\hline \multirow{2}{*}{$\begin{array}{l}\text { Fusion } \\
\text { Methods }\end{array}$} & & Green & Red & NIR & Mean \\
\hline & LISS-III & 0.87 & 0.86 & 0.75 & 0.83 \\
\hline \multirow{3}{*}{ Wavelet } & G-MX & 0.84 & 0.83 & 0.75 & 0.81 \\
\hline & R-MX & 0.84 & 0.84 & 0.74 & 0.80 \\
\hline & NIR-MX & 0.78 & 0.76 & 0.81 & 0.79 \\
\hline \multirow{3}{*}{$\mathrm{HPF}$} & G-MX & 0.88 & 0.81 & 0.87 & 0.85 \\
\hline & R-MX & 0.88 & 0.88 & 0.87 & 0.87 \\
\hline & NIR-MX & 0.87 & 0.65 & 0.86 & 0.79 \\
\hline \multirow{3}{*}{ HIS } & G-MX & 0.70 & 0.56 & 0.33 & 0.53 \\
\hline & R-MX & 0.66 & 0.57 & 0.35 & 0.53 \\
\hline & NIR-MX & 0.68 & 0.57 & 0.33 & 0.50 \\
\hline \multirow{3}{*}{ Brovey } & G-MX & 0.41 & 0.38 & 0.24 & 0.35 \\
\hline & R-MX & 0.49 & 0.48 & 0.07 & 0.35 \\
\hline & NIR-MX & 0.20 & 0.17 & 0.45 & 0.27 \\
\hline \multirow{4}{*}{ PCA } & G-MX & 0.51 & 0.25 & 0.44 & 0.40 \\
\hline & R-MX & 0.49 & 0.31 & 0.48 & 0.42 \\
\hline & NIR-MX & 0.20 & 0.39 & 0.25 & 0.28 \\
\hline & LISS-IV & 0.87 & 0.95 & 0.77 & 0.87 \\
\hline
\end{tabular}

Table-3: Q index for different fusion methods

\subsection{Visual Interpretation}

Visual interpretation of False Colour Composite (FCC) of the merged products revealed that R-MX was found to be superior across different fusion methods and depicted in Fig4. The PCA transformed image was of very poor quality and hence was not used in further analysis. Comparison of these images indicated that wavelet fusion method relatively retained spectral characteristics of $\mathrm{L}-3$ as compared to Brovey and PCA methods. The field boundaries and linear features were very sharp in case of wavelet and similar to original L-4MX data. The quality of PCA and Brovey transformed images was poor and the features were not clearly discernible due large changes in brightness. The 
different crop signatures on HPF and wavelet images was more or less comparable with original L-4MX data indicating that both spectral and spatial characteristics were preserved. Finally, classification accuracy of these transformed images was compared with the L-4 data for interpretability of different agricultural and horticultural crops.

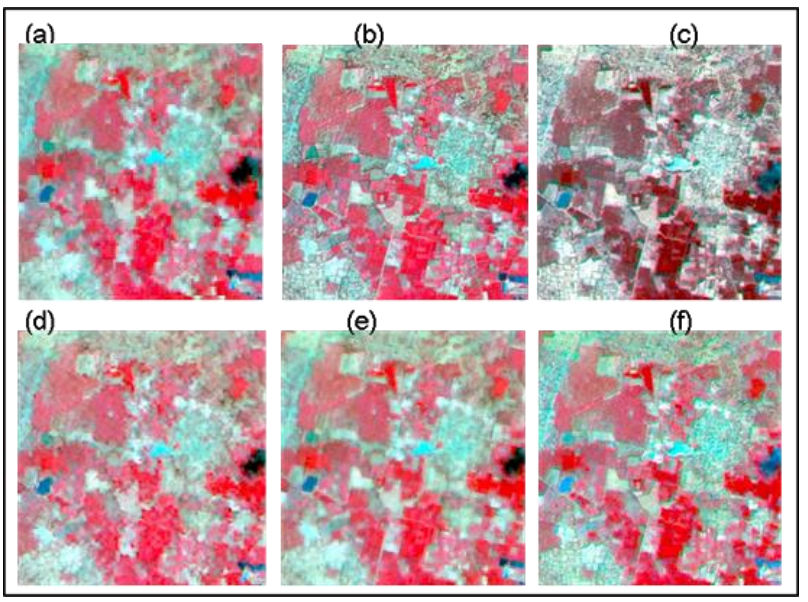

Fig-4: FCC images of (a) L-3 (b) L-4MX (c) Brovey d) IHS (e) HPF and (f) Wavelet

\subsection{Classification Accuracy}

The classification accuracies of L-3, L-4 and merged images were compared and the results are presented in Table-4. Rice and sugarcane crops were cultivated in large contiguous areas and thus, showing higher classification accuracy of above 95.0 per cent suggesting suitability of $24 \mathrm{~m} \mathrm{~L}-3$ data for discrimination. However, the horticultural crops showed poor classification accuracy using L-3 data alone. This can be attributed to spectral mixing of these crops due to sparse distribution and small holdings indicating that L-3 data at $24 \mathrm{~m}$ was unable to resolve these crops to obtain reasonable classification accuracy. The horticultural crops are more discernible in high resolution L-4 data due to sharpness of field boundaries. Class accuracies of smooth textured classes like rice and sugarcane was above 90 per cent while horticultural crops like oil palm and coconut showed accuracies close to 80 per cent as the these plantations are cultivated in contiguous area with closer spacing. The classification accuracy was poor for cashew as these were scattered throughout the study area with wider spacing and hence, per pixel classifier was not suitable for achieving reasonable accuracy.

\begin{tabular}{|l|r|r|r|r|}
\hline Classes & \multicolumn{1}{|c|}{ L-3 } & \multicolumn{1}{c|}{ L-4 } & Wavelet & \multicolumn{1}{c|}{ HPF } \\
\hline Rice & 99.2 & 97.9 & 98.1 & 94.7 \\
\hline Sugarcane & 97.1 & 87.9 & 97.1 & 90.1 \\
\hline Oil palm & 78.2 & 80.5 & 83.1 & 62.1 \\
\hline Coconut & 73.6 & 83.1 & 83.8 & 88.5 \\
\hline Cashew & 70.5 & 73.6 & 76.9 & 76.4 \\
\hline Fallow & 86.9 & 97.1 & 98.5 & 95.4 \\
\hline Water & 94.8 & 94.8 & 95.8 & 93.5 \\
\hline Urban & 95.4 & 90.8 & 84.1 & 82.1 \\
\hline OA & 84.3 & 89.7 & 92.2 & 82.7 \\
\hline Kappa & 0.82 & 0.87 & 0.90 & 0.79 \\
\hline
\end{tabular}

Table-4: Classification accuracy of different fusion products
Brovey transform showed poor classification accuracy due to lower dynamic range while the higher variance associated with PCA method resulted in poor classification accuracy. Due to radiometric distortions in Brovey and PCA, these methods are not recommended for the data fusion (Colditz et al., 2006). The overall classification accuracy of the wavelet merged data was 90 per cent in comparison to 79 per cent with that of the HPF method. The class accuracies for the three horticultural crops that had shown lesser accuracies individually with both L-3 and L-4 datasets improved in the wavelet merged product. The classification accuracy of wavelet merged product was on par with that of L-4 data indicating the spectral and spatial similarity of the two datasets. However, the accuracy of the urban settlements did not improve in the merged product due to the very high variance associated with this class. The study had indicated the potential use of merged data for improved classification of crops in a multi-cropped area by exploiting wider swath of L-4 mono data by selection of appropriate data fusion technique.

\subsection{CONCLUSIONS}

In this study, utility of multi-sensor data from Resourcesat-1 was evaluated for discrimination of crops using different data fusion techniques for selection of optimal monochromatic band of L-4MX data for merging with L-3 data. Performance of each band of L-4MX data for data fusion indicated that red band is most suitable for data fusion. The result confirms the specification of Resourcesat-1 wherein the L-4 mono mode nominally operates in the red band for achieving higher swath of about $70 \mathrm{~km}$.

Among the five methods evaluated, the wavelet method found to be superior for visual interpretability, image quality parameters and classification accuracy. The wavelet merged data was spectrally similar to L-3 data while maintaining spatial resolution of L-4 data enabling better discrimination of crops. The study suggests that wider swath of L-4 data in mono mode could be exploited by data fusion techniques in the absence of multispectral L-4MX data by acquiring panchromatic data in the red band. Further, investigations towards exploiting higher radiometry and spatial resolution of Cartosat series of satellites along with advanced classifiers using textural information needs to be addressed for improving classification accuracy in heterogeneous and multiple cropping systems.

\subsection{REFERENCES}

AS Field Guide, 2003. $7^{\text {th }}$ Edition, Leica Geosystems GIS \& Mapping, pp. 165-170.

Chavez, P. S., Sides, C. S. and Anderson, J. A., 1991. Comparison of three different methods to merge multiresolution and multispectral data: Landsat TM and SPOT panchromatic. PE \& RS, 57, pp. 295-303.

Chen Yunhao, Deng Lei, Li Jing, Li Xiaoping and Shi Peijun, 2006. A new wavelet-based image fusion method for remotely sensed data. International Journal of Remote Sensing, 27(7), pp.1465-1476.

Colditz, R.R., Thilo Wehrmann, Martin Bachmann, Klaus Steinnocher, Michael Schmidt, Gu Nter Strunz, and Stefan Dech, 2006. Influence of image fusion approaches on 
classification accuracy: a case study. International Journal of Remote Sensing, 27(15), pp. 3311-3335.

King, R.L. and Wang, J., 2001. A wavelet based algorithm for pan sharpening Landsat 7 Imagery. In IEEE Geosciences and Remote Sensing Symposium, IGARSS'01, 2, pp. 849851.

Kumar, A. S., Kartikeyan, B., and Majumdar, K. L. 2000. Band sharpening of IRS multispectral imagery by cubic spline wavelets. International Journal of Remote Sensing, 21, pp. 581-594.

Kumar, A.S., Kumar, A.S.K., Navalgund, R.R., 2006. Selection of IRS-P6 LISS-4 MO mode band for producing band-sharpened multispectral imagery. IEEE Geosciences and Remote Sensing Letters, 3 (1), pp. 32-35.

Mohanty K. K. and Majumdar T. J., 2003. Comparison of adaptive filter, IHS and Fourier transform based techniques for IRS-1C PAN/L-3 data merging. International Journal of Remote Sensing, 24(9), pp. 1969-1979.

Pohl, C., and Van Genderen, J. L., 1998. Multi-sensor image fusion in remote sensing: concepts, methods and applications. International Journal of Remote Sensing, 19, pp. $823-854$.

Ray, S.S., 2004. Merging of IRS LISS III and PAN dataevaluation of various methods for a predominantly agricultural area, International Journal of Remote Sensing, 25(13) 2657-2664.

Richards J.A. 1986. Remote Sensing Digital Image Analysis. Springer-Verlag Berlin Heidelberg.

Wald, L., Ranchin, T. And Mangolini, M., 1997. Fusion of satellite images of different spatial resolutions: assessing the quality of resulting images. PE \& RS, 63, pp. 691-699

Wang, Z. and Bovik, A.C., 2002. A universal image quality index. IEEE Signal Processing Letters, 9, pp. 81-84.

Wang, Z., Ziou, D., Armenakis, C., Li, D. and Li Q., 2005. A comparative analysis of image fusion methods. IEEE Transactions on Geosciences and Remote Sensing, 43, pp. $1391-1402$

Welch, R., and Ehlers, W., 1987. Merging multi-resolution SPOT HRV and Landsat TM data. Photogrammetric Engineering and Remote Sensing, 53, pp. 301-303. 